Reprod. Nutr. Dévelop., 1982, 22 (1 B), 243-249.

\title{
Influence du type d'innervation des fibres musculaires sur l'acquisition des propriétés de l'ATPase myofibrillaire
}

\author{
J. P. TOUTANT (1)
}

Laboratoire de Physiologie, Université de Nantes et Laboratoire de Neurobiologie, Ecole Normale Supérieure, 46, rue d'UIm, 75230 Paris Cedex 05.

Summary. Relationship between innervation and myofibrillar ATPase properties during development of chick muscles.

We present some observations differing from the idea that neurons precisely control the differentiation of muscle fibre histochemical properties and show that, in a few cases, innervation does not influence the development of myofibrillar ATPase properties. Moreover, we report that a regional variation of the acid stability of myofibrillar ATPase may occur in the polar zone of intrafusal fibres in chick PLD muscle spindles, a zone devoid of innervation. This observation is related to a similar variation in the polar zone of rat intrafusal fibres (Kucera et al., 1978). New examples are presented supporting the conclusion that the differentiation of the properties of ATPase activity, and thus of fibre types, takes place, at least in some cases, independently of the type of innervation received.

\section{Introduction.}

L'homogénéité des propriétés enzymatiques des fibres musculaires appartenant à une même unité motrice, suggère que le motoneurone contrôle le niveau d'activité de I'ATPase myofibrillaire (Edström et Kugelberg, 1968 ; Burke et al., 1971) et celui des enzymes du métabolisme oxydatif (Nemeth et al., 1980). Ce contrôle nerveux est également illustré par les résultats des expériences d'innervation croisée : le muscle soléaire de cobaye (muscle purement lent) réinnervé par un nerf étranger contenant des axones " rapides », présente 40 p. 100 de fibres musculaires " rapides" après six mois de réinnervation (Karpati et Engel, 1967).

Chez le poulet, les expériences de réinnervation croisée entre les muscles Grands Dorsaux Antérieur (ALD, lent) et Postérieur (PLD, rapide) n'induisent que des transformations limitées quand l'opération est pratiquée chez des animaux adultes (Hnik et al., 1967 ; Koenig, 1970 ; Koenig et Fardeau, 1973). Au contraire,

(1) Adresser toute correspondance à : J. P. Toutant, Laboratoire de Neurobiologie, E.N.S., 46, rue d'Ulm, 75230 Paris Cedex 05. 
réalisée chez des poussins peu de temps après l'éclosion, la réinnervation croisée induit la synthèse de myosine rapide dans le muscle ALD (Syrový et Zelená, 1975) et les fibres du muscle rapide (PLD) acquièrent des vitesses de contraction et une activité APTasique de type lent (Hnik et al., 1977). D'après Cosmos et al. (1979), le PLD réinnervé, à l'éclosion, par le nerf de l'ALD présente, 13 semaines après l'opération, certaines fibres dont les propriétés de l'ATPase ressemblent à celles des fibres de l'ALD. Enfin, pour Gordon et al. (1977), le muscle ALD régénérant à la place du PLD, et réinnervé par le nerf du PLD, présente les caractères histochimiques des fibres du PLD. Ces observations, ainsi que les résultats des expériences de stimulations musculaires chroniques (Salmons, 1980), suggèrent que la différenciation de I'ATPase myofibrillaire des fibres musculaires est sous la dépendance du type d'activité nerveuse.

Les fibres musculaires toniques des Oiseaux présentent une multi-innervation distribuée sur toute leur longueur et constituent, pour cette raison, un matériel intéressant pour l'étude d'une éventuelle détermination nerveuse de leurs propriétés histochimiques. En effet, une fibre d'ALD peut présenter jusqu'à 80 plaques motrices « en grappe " (Ginsborg et Mackay, 1961) et ces terminaisons appartiennent à plusieurs motoneurones (Bennett et Pettigrew, 1974 ; Vrbová et al., 1978). Au moment de l'excitation nerveuse, chaque terminaison motrice n'active qu'une zone limitée de la fibre musculaire par l'intermédiaire d'un potentiel non propagé (Vrbová et al., 1978). L'activité contractile d'origine nerveuse varie donc d'un niveau de la fibre tonique à l'autre et on pouvait se demander si les caractères de l'ATPase myofibrillaire ne variaient pas parallèlement à l'innervation. Or, ni les fibres toniques du muscle Biventer cervicis, ni celles de l'ALD ne présentent une quelconque variation longitudinale de leur profil histochimique, en dépit de la présence, sur les longueurs de fibres examinées, d'un grand nombre de terminaisons motrices (Ashmore et al., 1978 ; Toutant et al., $1981 \mathrm{~b}$; Rouaud et Toutant, 1982). Cette uniformité longitudinale de l'activité ATPasique des fibres toniques multi-innervées est donc comparable à celle des fibres phasiques focalement innervées (Pette et Schnez, 1977).

Néanmoins, il existe un cas où des variations longitudinales de l'activité ATPasique ont été mises en évidence dans une même fibre musculaire : il s'agit des zones polaires des fibres intrafusales de rat, où la résistance de l'activité ATPasique à la préincubation acide peut varier (de la labilité totale à la résistance complète). Ces variations longitudinales sont souvent sans rapport avec la localisation des terminaisons fusimotrices (Kucera et al., 1978). La figure représente le résultat d'une étude comparable portant sur les fuseaux neuro-musculaires de PLD de poulet, 8 semaines après l'éclosion.

FIG. 1. - Coupes transversales successives d'un fuseau neuromusculaire de PLD de poulet, 8 semaines après /'éclosion. ATPase myofibrillaire après préincubation acide. Le schéma du fuseau permet de localiser les différents niveaux des coupes : de a à $f:$ zone polaire (extracapsulaire) ; de $g$ à $h$ : zone juxtaéquatoriale (encapsulée) ; i : zone équatoriale (encapsulée). Les flèches indiquent une fibre intrafusale qui présente une transformation du profil histochimique entre le niveau $c$ (type $\beta$ ) et le niveau $f$ (type $\alpha$ ). $\alpha$ et $\beta$ : les deux types de fibres (intra et extrafusales). $N$ : nerf du fuseau avant $(h)$ et après (i) son entrée dans la zone équatoriale. Grossissements $: \times 580$ sauf pour h et $\mathrm{i}: \times 260$. 


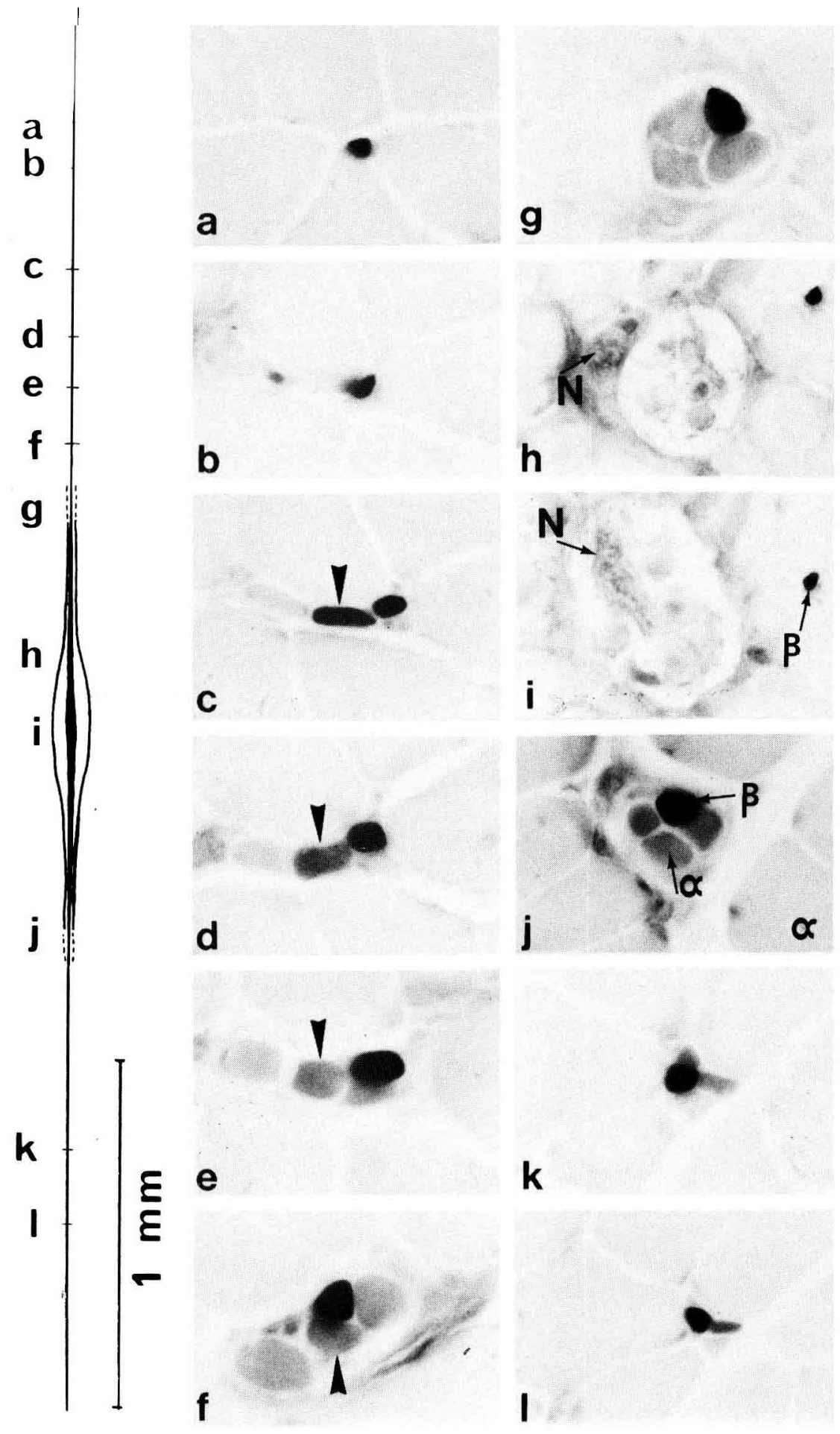

Reproduction, nutrition, développement $n^{\circ} 1 \mathrm{~B}$ 


\section{Matériel et méthodes}

Les muscles PLD sont prélevés, congelés et coupés transversalement au cryostat (épaisseur des coupes : $20 \mu \mathrm{m}$ ). Les coupes sont traitées pour la révéla-

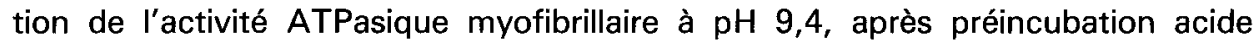
$(\mathrm{pH} 4,35)$ d'après la méthode de Guth et Samaha (1970). Les 200 coupes successives correspondant à la longueur totale du fuseau $(4 \mathrm{~mm})$ ont été examinées. Certaines de ces coupes sont représentées sur la figure avec l'indication de leur niveau longitudinal.

\section{Résultats.}

En se fondant sur la résistance de I'ATPase myofibrillaire à la préincubation acide, deux types principaux de fibres intrafusales peuvent être définis dans les zones juxtaéquatoriales des fuseaux (niveaux $\mathrm{g}$ et $\mathrm{j}$ de la figure). Les fibres dont l'ATPase est inhibée par la préincubation acide peuvent être comparées aux fibres extrafusales de type "fast-twitch " (type $\alpha$ ou II), largement majoritaires dans le muscle PLD. Les fibres intrafusales dont l'ATPase est résistante à la préincubation acide sont comparables aux fibres extrafusales de type "slow-tonic " (type $\beta$ ), également décrites dans le PLD, quoique en très petit nombre chez l'adulte (Koenig et Fardeau, 1973 ; Toutant et al., 1979). La figure montre également (entre les niveaux $\mathrm{c}$ et $\mathrm{f}$ ), une variation longitudinale des propriétés de I'ATPase myofibrillaire entre les différents niveaux de la zone polaire d'une fibre intrafusale : de type $\beta$ au niveau c, la fibre passe au type $\alpha$ aux niveaux e et $f$. Cette variation régionale du profil histochimique a été observée dans plusieurs zones polaires de fibres intrafusales. D'après Chin (1970), l'innervation fusimotrice $\gamma$ des fibres intrafusales du PLD de poulet est limitée à la zone juxtaéquatoriale, capsulaire, correspondant aux niveaux $\mathrm{g}$ et $\mathrm{j}$ de la figure. La variation d'activité ATPasique, notée ci-dessus dans la zone polaire, se produirait donc dans une zone dépourvue d'innervation. Cette conclusion est en accord avec celle de Kucera et al. (1978), déjà citée.

\section{Discussion.}

D'autres observations viennent étayer le résultat précédent, et suggèrent que la différenciation de l'activité ATPasique des fibres musculaires intra ou extrafusales pourrait être indépendante du contrôle nerveux.

Au cours du développement embryonnaire des fuseaux neuromusculaires du PLD, deux types histochimiques de fibres intrafusales $(\alpha$ et $\beta$ ) sont observés dès que les fuseaux eux-mêmes apparaissent lau 14e jour de l'incubation pour le PLD). Or, à ce moment du développement, les caractères morphologiques ultrastructuraux des terminaisons fusimotrices sont très primitifs (Toutant et al., 1981 a) et les fuseaux ne sont pas fonctionnels (Narayanan et Malloy, 1974). Par 
ailleurs, les fuseaux neuromusculaires de PLD de poussins contiennent de 1 à 8 fibres intrafusales, et à partir de 5 fibres, 1 fibre $\beta$ au moins, est observée parmi les fibres $\alpha$ toujours majoritaires ; l'étude du nerf entrant dans la zone équatoriale de plusieurs fuseaux a montré que quelques fuseaux ayant plus de 4 fibres sont innervés par une fibre la et un seul axone $\gamma$. Dans ce cas il paraît peu vraisemblable que le même axone $\gamma$ puisse induire la différenciation de l'activité ATPasique des fibres qu'il innerve vers deux types histochimiques différents (types $\alpha$ et $\beta$ ) (Toutant et al., 1981a).

D'autre part, le résultat suivant souligne, lui aussi, une relative indépendance de la différenciation histochimique des ébauches musculaires vis-à-vis de l'innervation : la zone de tube neural correspondant à l'innervation présomptive des muscles Grands Dorsaux est remplacée chez l'embryon de poulet au stade 22 somites par un segment tout à fait postérieur (lombaire) de tube neural prélevé sur un autre embryon de même stade. Cette profonde modification de la nature des motoneurones assurant l'innervation des deux muscles se traduit par une transformation du " pattern » synaptique des fibres musculaires extrafusales, mais les propriétés de l'ATPase myofibrillaire des deux muscles (prélevés entre le $16^{\mathrm{e}}$ et le $18^{\mathrm{e}}$ jour de l'incubation) sont très peu affectées par l'opération : les muscles ALD et PLD restent très facilement identifiables d'après ce critère (Khaskiye et al., 1980).

Enfin, on peut formuler quelques remarques sur les résultats des expériences de réinnervation croisée et de greffe rapportées dans l'introduction de cet article. Les résultats de Gordon et al. (1977), qui concernent le muscle ALD régénérant à la place du PLD et réinnervé par le nerf de ce dernier, et ceux de Cosmos et al. (1979), décrivant l'effet de la réinnervation croisée du PLD par le nerf de I'ALD, montrent une transformation significative du type histochimique des fibres musculaires. Néanmoins, Cosmos souligne que le pourcentage de fibres affectées par l'opération varie de façon importante d'un animal à l'autre. De plus, l'examen attentif des fibres transformées montre que les fibres "toniques" apparaissant dans le PLD après réinnervation par le nerf de l'ALD ne sont pas tout à fait assimilables à l'un des types de fibres du muscle ALD témoin. Ces fibres transformées se rapprocheraient plutôt des rares fibres de type $\beta$ observées dans le PLD témoin. Cette remarque s'applique également aux résultats de Gordon : si le muscle ALD régénérant à la place du PLD présente bien des fibres $\alpha$ comparables à celles du PLD, le muscle présente aussi un pourcentage anormalement élevé de fibres $\beta$. Ces deux remarques permettent de citer la conclusion de Ashmore et Doerr (1976), qui, greffant un muscle ALD à la place du Biceps brachii (muscle rapide), observent une conversion incomplète des types de fibres, le greffon se caractérisant par un pourcentage élevé de fibres $\beta$ dont l'ATPase résiste à la préintubation acide, alors que le Biceps présente 99 p. 100 de fibres labiles $(\alpha)$ et que le PLD greffé dans les mêmes conditions ne présente que des fibres $\alpha$. Les auteurs concluent à une dominance incomplète du nerf sur le muscle, le phénotype exprimé par les cellules musculaires pendant l'embryogenèse résistant à l'influence d'un nerf étranger, même après plusieurs mois.

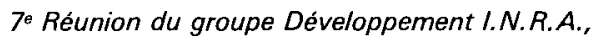
Nouzilly/Tours, 14-15 mai 1981. 


\section{Références}

ASHMORE C. R., DOERR L., 1976. Transplantation of the anterior latissimus dorsi muscle of the chicken. Exp. Neurol., 30, 312-318.

ASHMORE C. R., KIKUCHI T., DOERR L., 1978. Some observations on the innervation of different fiber types of chick muscle. Exp. Neurol., 58, 272-284.

BENNETT M. R., PETTIGREW A. G., 1974. The formation of synapses in striated muscle during development. J. Physiol. (Lond.), 241, 515-545.

BURKE R. E., LEVINE D. N., ZAJAC F. E., TSAIRIS P., ENGEL W. K., 1971. Mammalian motor units : physiological-histochemical correlation in three types in cat gastrocnemius. Science, 174, 709-712.

CHIN N. K., 1970. The structure and innervation of the muscle spindle in the latisimus dorsi anterior and posterior muscles of the domestic fowl. Ph. D. Thes. Univ. Hong Kong.

COSMOS E., BUTLER J., ALLARD E. P., MAZLIAH J., 1979. Factors that influence the phenotypic expression of genetically normal and dystrophic muscles. Ann. N. Y. Acad. Sci., 317, 571593.

EDSTRÖM L., KUGELBERG E., 1968. Histochemical composition, distribution of fibers and fatiguability of single motor units. J. Neurol. Neurosurg. Psychiat., 31, 423-433.

GINSBORG B. L., MACKAY B., 1961. A histochemical demonstration of two types of motor innervation in avian skeletal muscle. Biblioth. Anat., 2, 174-181.

GORDON T., PERRY R., SRIHARI T., VRBOVÁ G., 1977. Differentiation of slow and fast muscles in chickens. Cell Tiss. Res., 180, 211-222.

GUTH L., SAMAHA F. J., 1970 . Procedure for the histochemical demonstration of actomyosin ATPase. Exp. Neurol., 28, 365-367.

HNIK P., JIRMANOVÁ I., SYRIVÝ I., 1977. Functional transformation of fast posterior lattisimus dorsi muscle by "slow" nerve implanted in newly hatched chickens. Physiol. Bohemoslov., 26. 103-110.

HNIK P., JIRMANOVÁ I., VYKLICKY L., ZELENÁ J., 1967. Fast and slow muscles of the chick after nerve cross-union. J. Physiol. (Lond.), 193, 309-325.

KARPATI G., ENGEL W. K., 1967. Transformation of the histochemical profile of skeletal muscle by " foreign " innervation. Nature, 215, 1509-1510.

KHASKIYE A., TOUTANT J. P., TOUTANT M., RENAUD D., LE DOUARIN G., 1980 . Effect of heterotopic innervation on the development of synaptic pattern in chick embryo muscles. Arch. Anat. Microsc. Morph. exp., 69, 135-145.

KOENIG J., 1970. Contribution à l'étude des plaques motrices des Grands Dorsaux antérieur et postérieur du poulet après innervation croisée. Arch. Anat. Microsc. Morph. exp., 59, 403-426.

KOENIG J., FARDEAU M., 1973. Etude histochimique des muscles Grands Dorsaux antérieur et postérieur de poulet et des modifications observées après dénervation et réinnervation homologue ou croisée. Arch. Anat. Microsc. Morph. exp., 62, 249-267.

KUCERA J., DOROVINI-ZIS K., ENGEL W. K., 1978. Histochemistry of rat intrafusal muscle fibers and their innervation. J. Histochem. Cytochem., 26, 973-988.

NARAYANAN C. H., MALLOY R. B., 1974. Deafferentation studies on motor activity in the chick. II. Activity pattern of wings. J. exp. Zool., 189, 177-188.

NEMETH P., PETTE D., VRBOVÁ G., 1980. Malate dehydrogenase activity indicating metabolic homogeneity of single fibres of the motor unit. J. Physiol. (Lond.), 301, 73-74P.

PETTE D., SCHNEZ U., 1977. Myosin light chain patterns of individual fast and slow-twitch fibres of rabbit muscles. Histochemistry, 54, 97-107.

ROUAUD T., TOUTANT J. P., 1982. Histochemical properties and innervation pattern of fast and slox-tonic fibre types of the anterior latissimus dorsi muscle of the chick. Histochem. J., 14 (sous presse). 
SALMONS S., 1980. Functional adaptation in skeletal muscle. Trends neurosci, 3, 134-137.

SYROVY I., ZELENÁ J., 1975. The onset and progress of transformation of avian slow into fast muscles under neural influence. Pflügers Arch., 360, 121-134.

TOUTANT M., BOURGEOIS J. P., ROUAUD T., TOUTANT J. P., 1981 a. Morphological and histochemical differentiation of intrafusal fibres in the Posterior latissimus dorsi muscle of the developing chick. Anat. embryol., 162, 325-342.

TOUTANT J. P., ROUAUD T., LE DOUARIN G., 1981 b. Histochemical properties of the biventer cervicis of the chick : a relationship between multiple innervation and slow-tonic fibre types. Histochem. J., 13, 481-493.

TOUTANT J. P., TOUTANT M., RENAUD D., LE DOUARIN G., 1979. Enzymatic differenciation of muscle fibre types in embryonic latissimus dorsii of the chick : effects of spinal cord stimulation. Cell Differ., 8, 375-382.

VRBOVA G., GORDON T., JONES R., 1978. Nerve-muscle interaction. Chapman \& Hall, London. 